\title{
3D acoustic wave simulation using BEM formulations and the ACA algorithm
}

\author{
Jinlong Feng ${ }^{1}$, Xiaoping Zheng ${ }^{1}$, Haitao Wang ${ }^{2}$, HongTao Wang ${ }^{2}$, \\ Yuanjie Zou ${ }^{3}$, Yinghua Liu ${ }^{1} \&$ Zhenhan $\mathrm{Yao}^{1}$ \\ ${ }^{I}$ Department of Engineering Mechanics, Tsinghua University, \\ Beijing, China \\ ${ }^{2}$ Institute of Nuclear and New Energy Technology, Tsinghua University, \\ Beijing, China \\ ${ }^{3}$ China Academy of Space Technology, Beijing, China
}

\begin{abstract}
In this paper some applications of boundary element method are given to solve $3 \mathrm{D}$ acoustic problems in an exterior domain. The Burton-Miller technique is also used to ensure the uniqueness of solutions. In order to improve efficiency, Adaptive Cross Approximation is used to deal with both the system matrix and the vector, and the GMRES algorithm is adopted as the iteration solver. Three examples are presented to illustrate the effectiveness and accuracy of the method. Keywords: acoustics problems, Boundary Element Method, Adaptive Cross Approximation, GMRES algorithm.
\end{abstract}

\section{Introduction}

Acoustics problem is very important in many fields, such as automotive industry, high-speed train transport service, wind-generated systems, navigation, aerospace engineering and so on [1-2]. In the aerospace industry, for instance, spacecraft structures are subject to heavy acoustic load, particularly during launch, which can impose severe and adverse affect to the structures of the spacecraft and their payloads. The importance of the launcher vibroacoustic environment is increasing with respect to satellite loads due to the increase in size and decrease in surface mass of lightweight appendages like antennas and solar arrays. In this framework, the effect of acoustic loads to structures will be even more important in the course of the next decades with the introduction of 
new light-weight materials such as composites [3]. Several techniques have been developed [4-6], but the most conventional and efficient methods for engineering analyses are the Finite Element Method (FEM) and the Boundary Element Method (BEM).

The FEM is the preferred tool for the simulation of structural vibrations [7]. It can also be employed for the acoustic part; however, comparing with the BEM, the latter reduce by one the mathematical dimension of problems to be solved, from $3 \mathrm{D}$ to $2 \mathrm{D}$, or from $2 \mathrm{D}$ to $1 \mathrm{D}$, leading to much easier mesh generation. The second advantage is the accuracy the BEM offers, due to the nature of integrals used in the formulations. The third advantage of the BEM is its capability of automatic satisfaction of the Sommerfeld radiation condition [8] without introducing additional, often cumbersome, conditions at infinity.

On the contrary, boundary element matrices are non-symmetric and fully populated. Typically direct solvers require $\mathrm{O}\left(N^{3}\right)$ operations while iterative solvers $\mathrm{O}\left(\mathrm{k} N^{2}\right)$, where $\mathrm{k}$ is the number of iterations. The above drawbacks reduce the efficiency of the BEM. In this regard, the Adaptive Cross Approximation (ACA) $[9,10]$ algorithm is used to reduce the memory storage requirements and the CPU solution time. When come to iteration, the Generalized Minimal Residual Method (GMRES) iterative solver is adopted [11, 12].

The paper is organized as follows. After a short introduction to the subject, the Boundary Element Method for the Helmholtz equation is given. In Section 3, the adaptive cross approximation method and GMRES are covered. Numerical examples are given in Section 4. The last parts are conclusions and acknowledgements.

\section{The boundary elements method for the Helmholtz equation}

\subsection{Helmholtz equation}

We consider the linear time-harmonic acoustic wave problems in an exterior domain E, outside a structure with boundary S. Acoustic problems in frequency domain can be described by the Helmholtz equation, in three dimensions, which is written as

$$
\nabla^{2} p(x)+k^{2} p(x)=0
$$

where $\nabla^{2}$ is the Laplace operator, $p(\boldsymbol{x})$ is the sound pressure at point $\boldsymbol{x}$ in the fluid, $k=\omega / c$ is the acoustic wavenumber and $\mathrm{c}$ is the speed of sound in the fluid. Additionally the Sommerfeld radiation condition [8] in three dimensions

$$
\left|\frac{\partial p}{\partial r}-\mathrm{i} k p\right| \leq \frac{c}{r^{2}}, \quad r \rightarrow \infty
$$

has to be satisfied for this exterior problem, which ensures that wave is purely outgoing.

A 3D fundamental solution for the Helmholtz equation is given by

$$
G^{*}(x, y)=\frac{\mathrm{e}^{\mathrm{i} k r}}{4 \pi r}
$$


where $x$ is a field point, $y$ is a source point and $r=|x-y|$ is the distance between $x$ and $y$. The key idea is to use Green's second identity in combination with the property of the Dirac distribution.

$$
\int_{E}\left(p \nabla^{2} G^{*}-G^{*} \nabla^{2} p\right) \mathrm{d} V=\int_{S}\left(p \frac{\partial G^{*}}{\partial n}-G^{*} \frac{\partial p}{\partial n}\right) \mathrm{d} S
$$

By this way, the pressure $p$ at an arbitrary point $x$ within the exterior acoustic domain $\mathrm{E}$ is given by the integral representation

$$
p(x)=-\int_{S} G^{*}(x, y) q(y) \mathrm{d} S+\int_{S} \frac{\partial G^{*}(x, y)}{\partial n_{y}} p(y) \mathrm{d} S, \quad x \in \Omega^{\mathrm{E}}
$$

For scattering problems formulation (6) can be written

$$
p(x)=-\int_{S} G^{*}(x, y) q(y) \mathrm{d} S+\int_{S} \frac{\partial G^{*}(x, y)}{\partial n_{y}} p(y) \mathrm{d} S+p^{\mathrm{I}}(x), \quad x \in \Omega^{\mathrm{E}}
$$

Let the point $x$ approach the boundary. We obtain the following conventional boundary integral equation (CBIE) [13]:

$$
C(x) p(x)=\int_{S}\left(G^{*} \frac{\partial p}{\partial n}-p \frac{\partial G^{*}}{\partial n}\right) \mathrm{d} S
$$

where $C(x)=0.5$ if $S$ is smooth around $x$. This CBIE can be employed to solve for the unknown $p$ and $q$ on $S$.

It is well known that the CBIE has a major defect for exterior problems, that is, it has non-unique solutions at a set of fictitious eigenfrequencies associated with the resonance frequencies of the corresponding interior problems [14]. This difficulty is referred to as the fictitious eigenfrequency difficulty. A remedy to this problem is to use the normal derivative BIE in conjunction with the CBIE. Taking the derivative of integral representation (8) with respect to the normal at a point $x$ on $S$ and letting $x$ approach $S$, we obtain the following hypersingular boundary integral equation (HBIE):

$$
C(x) q(x)=-\int_{S} \frac{\partial G^{*}(x, y)}{\partial n_{x}} q(y) \mathrm{d} S+\int_{S} \frac{\partial^{2} G^{*}(x, y)}{\partial n_{x} \partial n_{y}} p(y) \mathrm{d} S, \quad x \in S
$$

where $C^{\prime}(x)=\frac{1}{2}$, if $S$ is smooth around $x$. This equation, taken by itself, suffers from the same kind of defect as equation (8). However, if we use a linear combination of CBIE (Eq. (8)) and HBIE (Eq. (9)), the uniqueness can be ensured for exterior acoustic wave problems. Thus, we obtain

$$
\begin{aligned}
& \int_{S} \frac{\partial G^{*}}{\partial n_{y}} p(y) \mathrm{d} S+C(x) p(x)+\alpha \int_{S} \frac{\partial^{2} G^{*}}{\partial n(x) \partial n(y)} p(y) \mathrm{d} S \\
& =\int_{S} G^{*} \frac{\partial p}{\partial n} \mathrm{~d} S+\alpha\left[\int_{S} \frac{\partial G^{*}}{\partial n_{x}} \frac{\partial p}{\partial n} \mathrm{~d} S-C^{\prime}(x) \frac{\partial p}{\partial n_{x}}(x)\right]
\end{aligned}
$$

where $\alpha$ is the coupling constant. This formulation is called Burton-Miller formulation [15] for acoustic wave problems and has been shown to yield unique 
solutions at all frequencies, if $\alpha$ is a complex number which, for example, can be chosen as $\alpha=\frac{\mathrm{i}}{\kappa}$ with $\mathrm{i}=\sqrt{-1}$ [14].

When the boundary of the acoustic domain is discretized into the boundary elements, the resulting system of linear equations can be expressed as

$$
[H][p]=[G][v]
$$

where the matrices $[\boldsymbol{H}]$ and $[\boldsymbol{G}]$ are obtained by integrating the fundamental solutions over each boundary element. When there is incident sound, a free term is needed and equation (11) becomes

$$
[\boldsymbol{H}][\boldsymbol{p}]=[\boldsymbol{G}][\boldsymbol{v}]+\left[\boldsymbol{p}_{I}\right]
$$

\section{Solving algorithm of BEM equations}

Because of non-symmetric and fully populate matrices in BEM equations, the Adaptive Cross Approximation (ACA) algorithm is used to compress and storage matrix entries and to compute matrix-vector multiplication. When it comes to iteration, the Generalized Minimal Residual Method (GMRES) iterative solver is adopted. In the following, the ACA and GMRES algorithm are introduced briefly. The details and more efficient version can be found in literature $[9,10,16]$.

\subsection{Adaptive cross approximation}

The Adaptive Cross Approximation algorithm produced by Bebendorf and Rjasanow is an effective technique for solving non-symmetric and fully populated matrices and decreases the CPU time significantly. The main process is as follows.

\section{1) Index octree}

The low-rank approximation of the matrix of BEM is based on asymptotically smooth functions, which happens in a well separated domain. Thus a tree structure for all boundary points should be constructed at first. For a 3D domain, an octree is necessary to describe the geometry relationship of sections on surface. The information that a tree should record includes: its father, its children, its geometry information, the points contained and so on. The least nodes are called leaves whose number of boundary points contained are less than a given threshold value used to judge if a new division is necessary.

\section{2) Partitioning of matrix}

Based on the tree, the matrix of BE equations can be divided into some blocks. At first, the relationship between nodes should be established. For some node NP in an octree, its neighbor nodes are defined as the nodes which have the same geometry size and one common point at least. The interaction nodes are those nodes owing the same geometry size, whose fathers are neighbour nodes, but not for them. The relationship is shown as Figure 1 in a two-dimension domain. 


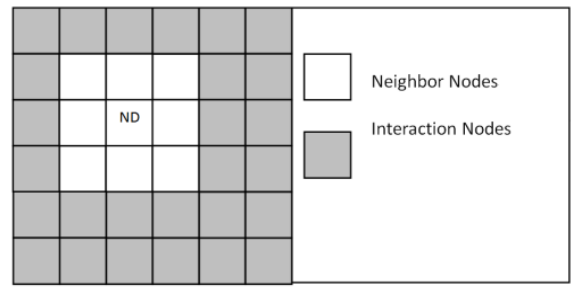

Figure 1: Relationship between nodes.

Then for every node of the tree, the elements contained are taken as source points and the elements of its neighbor as field points, and the sub matrix came from the source points and field points are defined $A_{n}$. The sub-matrices whose entries come from elements of the node and its interaction nodes are defined $A_{f}$. All the sub-matrices $A_{n}$ and $A_{f}$ form the whole efficient matrix of the boundary element equation.

\section{3) Low rank approximation}

The low-rank approximation in ACA is defined as

$$
\tilde{\boldsymbol{A}}^{m \times n}=\boldsymbol{U}^{m \times r} \boldsymbol{V}^{r \times n}=\sum_{i=1}^{r} \boldsymbol{u}_{i}^{m \times 1} \boldsymbol{v}_{i}^{1 \times n}
$$

The goal of ACA is to achieve

where $\varepsilon$ is the admissible error.

$$
\left\|\boldsymbol{R}^{m \times n}\right\|=\left\|\boldsymbol{A}^{m \times n}-\tilde{\boldsymbol{A}}^{m \times n}\right\| \leq \varepsilon\left\|\boldsymbol{A}^{m \times n}\right\|
$$

The ACA algorithm has been applied to the low rank blocks achieving approximately $\mathrm{O}(N)$ for both storage and matrix-vector multiplication [17]. It must be noted that the full pivoted approach is well known to be much slower than partially pivoted approach. The main reason behind this is that fully pivoted approach requires the knowledge of the full matrix whereas the partially pivoted approach would only require generation of individual matrix entries.

\subsection{Generalized minimal residual method (GMRES)}

The generalized minimal residual method (GMRES) is one of the most popular iterative solvers for asymmetrical linear systems proposed by Saad and Schultz [18] and further developed by other authors [19-21]. It has the property of minimizing at every step the norm of the residual vector over a Krylov subspace. The algorithm is derived from the Arnoldi process for constructing an orthogonal basis of Krylov subspace. Because of existence of error the gained vectors lose orthogonality gradually. Therefore a preconditioner is needed. In our codes the solution of diagonal blocks are taken as the initial solution and the maximal iteration time is set to 60 .

\section{Numerical results}

In this section the numerical results of three different examples of simulations are given. The first regards the results of a simple benchmark problem whose 
analytical solution is well known [22], i.e., a uniform pulsating sphere. The second example evaluates the pressure distribution on the surface and in the surroundings of a rigid sphere. The third example simulates the pressure distribution on the surface and in the surroundings of a satellite suffering incident plane wave. In all the examples constant triangular elements are utilized to discretize the problems. The maximum number of elements in a leaf is set to 50. The optimum value of this parameter depends upon the geometry and the elements of the mesh. The GMRES solver will stop iterations when the residue is below the tolerance $10^{-8}$. All the computations were done on a desktop PC with an Intel $3.30 \mathrm{GHz}$ processor and $4.00 \mathrm{G}$ memory.

\subsection{The sound generated by a pulsating sphere}

The problem of the sound radiated by a pulsating sphere with radius $r=1 \mathrm{~m}$, and uniform radial velocity $v_{n}=1 \mathrm{~m} / \mathrm{s}$ is investigated. The acoustic wave velocity and the medium density are set equal to unity. The wavenumber $k$ varies from 1 to 20 . The total number of elements is 800 . This surface vibration will generate the centred wave and the analytical solution of this problem is

$$
p(r)=\frac{\mathrm{i} k}{\mathrm{i} k-1} Z_{0} v_{n}
$$

where $Z_{0}=\rho_{0} c$ is the acoustic impedance.

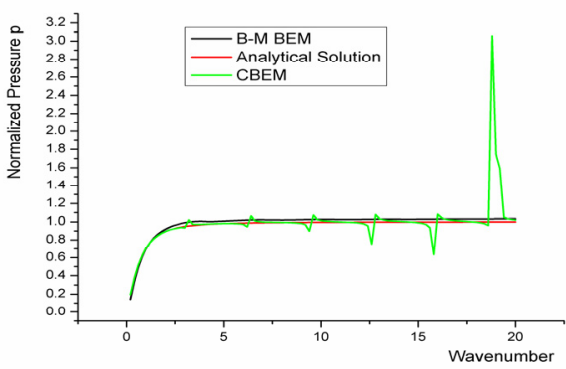

Figure 2: Frequency sweep plot for the pulsating sphere model.

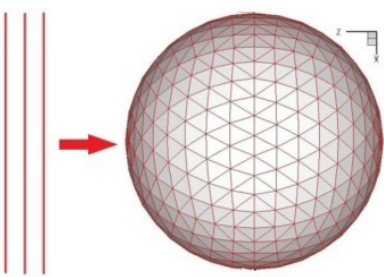

Figure 3: $\quad$ Plane wave scattered by a rigid sphere.

The three-dimensional scalar wave propagation problem using constant boundary elements is used for comparison purposes. The results from CBEM, Burton-Miller method and analytical solution are compared in Figure 2, which shows that the conventional BEM fails to predict the pressure on the surface of the sphere at the fictitious frequencies. The results from the Burton-Miller formulation compare well with the analytical solution at all wave numbers. 


\subsection{Scattering of sound from a rigid sphere}

The second example is the scattering of sound from a rigid sphere of radius $r=1 \mathrm{~m}$ due to an incident plane wave coming from the $+z$ direction at different values of ka.

A plane wave of amplitude $p_{0}=1$ propagating in the direction of $+z$ direction is scattered by the rigid sphere centered at the origin $(0,0,0)$. The sphere in the presence of the plane wave is shown in Figure 3.

An incident plane wave of amplitude $p_{0}$ traveling in $+z$ direction is given by

$$
p_{\text {inc }}=p_{0} e^{\mathrm{i} z z}
$$

The total sound pressure at any field point is the sum of the incident and scattered pressures,

$$
p_{\text {total }}=p_{\text {inc }}+p_{\text {scattered }}
$$

A BEM model consisting of 402 nodes and 800 constant triangular elements was used to model the sphere.

Figure 4 shows angular distribution of the field point pressure amplitudes at $r=1.5$ computed from both BEM and analytical methods for different values of

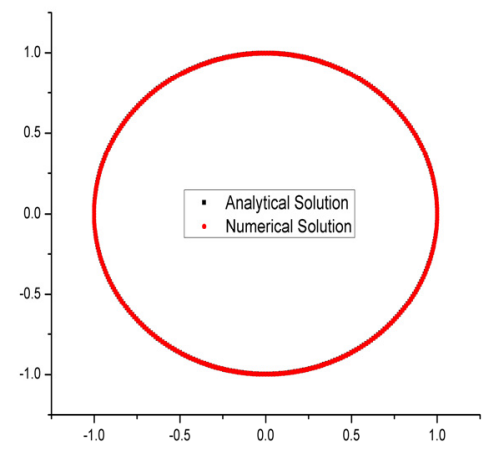

(a)

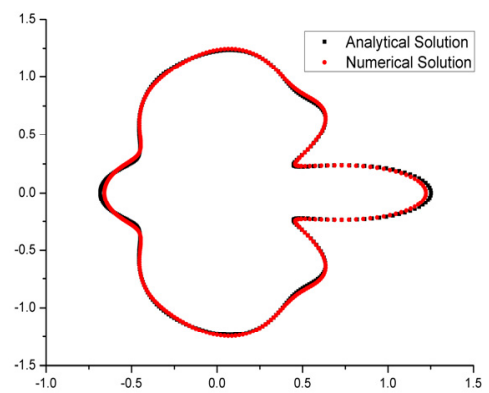

(c)

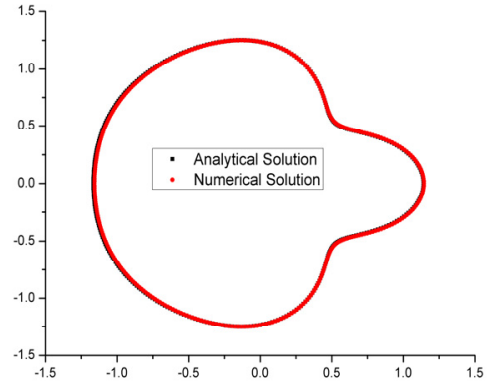

(b)

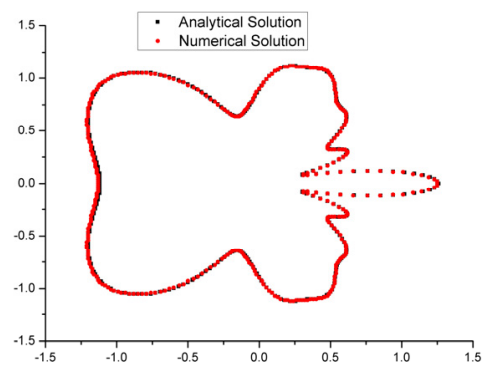

(d)

Figure 4: Angular distribution of the field point pressure for different values of $k a$. (a) $k a=0.1$, (b) $k a=2$, (c) $k a=4$, (d) $k a=8$. 
$k a$. The quantities are plotted versus the polar angle $\theta \cdot \theta=\pi$ corresponds to the front end and $\theta=0$ corresponds to the back end of the sphere with respect to the impinging plane wave. The comparisons between the solutions computed from BEM and analytical expressions show very good agreement.

\subsection{Scattering of sound from a satellite}

Another example is to simulate the pressure distribution on the surface and in the surroundings of a satellite as shown in Figure 5.

A spherical wave, whose frequency is $80 \mathrm{~Hz}$ and sound velocity is $c=344 \mathrm{~m} / \mathrm{sec}$, is used to simulate the real vibration experiment. Figure 6 shows the pressure distribution on the surface of the satellite and in Figure 7 there is the pressure distribution surrounding it.

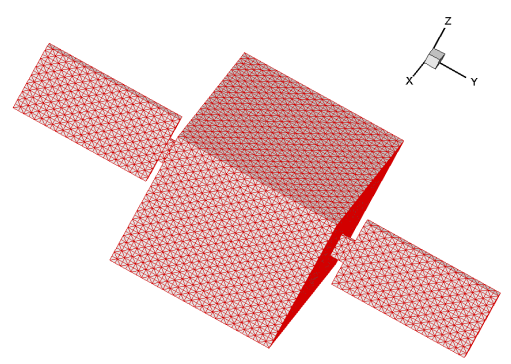

Figure 5: $\quad$ Surface of a satellite meshed with 13380 triangular elements.

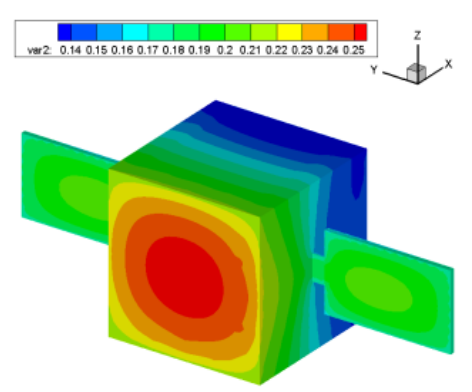

Figure 6: $\quad$ Sound pressure for a satellite.

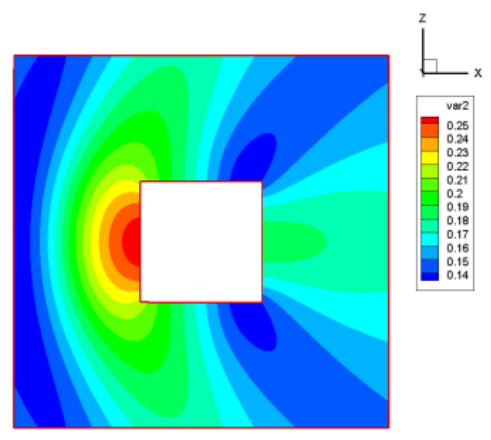

Figure 7: $\quad$ Sound pressure surrounding a satellite.

\section{Conclusion}

The BEM has successfully predicted the acoustic radiation and scattering in an infinite domain and the numerical results agree with the analytical solutions very 
well. After verifying the accuracy of the formulation and codes, we compare the efficiency of conventional BEM with the ACA BEM. The latter enhance computing efficiency greatly. Then a complex satellite model suffering a plane wave is evaluated, which indicates that it's a potential method to solve large scale acoustic problems with ACA algorithm.

\section{Acknowledgement}

The work is jointly supported by the National Natural Science Foundation of China (No.11272182 and No. 11072128).

\section{References}

[1] Brancati, A., Aliabadi, M.H., Milazzo, A. An Improved Hierarchical ACA Technique for Sound Absorbent Materials. CMES, 2011, 78(1): 1-24.

[2] Yijun Liu, Milind Bapat. Fast multipole boundary element method for 3-d full- and halfspace acoustic wave problems. IMECE2009-10165, November 13-19, Lake Buena Vista, Florida, USA.

[3] Erwin Perl, Thinh Do, Alan Peterson, John Welch. Environmental testing for launch and space vehicles [J]. Crosslink, 2005.

[4] Gladwell, G.M.L., Zimmermann, G. On energy and complementary energy formulations of acoustic and structural vibration problem [J]. Journal of sound and vibration, 1966, 3(3): 233-241.

[5] Smith, R.R., Hunt, J.T. and Barach, D. Finite element analysis of acoustically radiating structures with applications to sonar transducers [J]. Journal of sound and vibration, 1973, 54(5): 1277-1288.

[6] Zienkiewicz, O.C., Bando, K. et al. Mapped infinite elements for exterior wave problems [J]. International Journal for Numerical Methods in Engineering, 1985, 21(7): 1229-1251.

[7] Zienkiewicz O.C., Taylor R.L. The finite element method, vols. 1, 2.4th ed. London: McGraw-Hill; 1991.

[8] Sommerfeld A., Partial differential equations in physics, New York., academic press. pp. 256-344 (1949).

[9] Bebendorf M. Application of boundary element matrices. Numer. Math., 2000; 86: 565-589.

[10] Bebendorf M. Adaptive low-rank approximation of collocation matrices. 2003; 70: 1-24.

[11] Von Estorff, O., Rjasanow, S., Stolper, M., Zalesk, O. (2005): Two efficient methods for a multifrequency solution of the Helmholtz equation. Computing and Visualization in Science, vol. 8, pp. 159-167.

[12] Generalized minimal residual method. Wikipedia, the Free Encyclopedia. http://en.wikipedia.org/wiki/Generalized_minimal_residual_method.

[13] Seybert, A.F., Soenarko, B., Rizzo, F.J., and Shippy, D.J., 1985, “An Advanced Computational Method for Radiation and Scattering of Acoustic Waves in Three Dimensions,” J. Acoust. Soc. Am., 77, pp. 362-368. 
[14] Kress, R., 1985, "Minimizing the Condition Number of Boundary Integral Operators in Acoustic and Electromagnetic Scattering," Quart. J. Mech. Appl. Math., 38, pp. 323-341.

[15] Burton, A.J., and Miller, G.F., 1971, "The Application of Integral Equation Methods to the Numerical Solution of Some Exterior Boundary-Value Problems," Proc. R. Soc. London, Ser. A, 323, pp. 201-210.

[16] Von Estorff, O., Rjasanow, S., Stolper, M., Zalesk, O. (2005): Two efficient methods for a multifrequency solution of the Helmholtz equation. Computing and Visualization in Science, vol. 8, pp. 159-167.

[17] Benedetti, I., Aliabadi, M.H., Daví G. (2007): A fast 3D dual boundary element method based on hierarchical matrices. International Journal of Solids and Structures, vol. 45, no. 7-8, pp. 2355-2376.

[18] Saad, Y., Schultz, M.H. (1986): GMRES: A generalized minimal residual algorithm for solving nonsymmetric linear systems. SIAM J. Sci. Stat. Comput., vol. 7, no. 3, pp. 856-869.

[19] Leung, C.Y., Walker, S.P. (1997): Iterative solution of large three dimensional BEM elastostatic analyses using the GMRES technique. International Journal for Numerical Methods in Engineering, vol. 40, pp. 2227-2236.

[20] Merkel, M., Bulgakov, V., Bialecki, R., Kuhn, G. (1998): Iterative solution of large-scale 3D-BEM industrial problems. Engineering Analysis with Boundary Elements, vol. 2, pp. 183-197

[21] Amini, S., Maines, N.D. (1998): Preconditioned Krylov Subspace methods for Boundary Element solution of the Helmholtz Equation. International Journal for Numerical Methods in Engineering, vol. 41, pp. 875-898.

[22] Pierce, A.D. (1981): Acoustic: an introduction to its Physical Principles and Applications. McGraw Hill, New York.

[23] Rayleigh, J.W.S. The Theory of Sound - Volume II. Dover, 1945. p. 272. 